\title{
Article \\ Optimal Modeling of an Elevator Chassis under Crash Scenario Based on Characterization and Validation of the Hyperelastic Material of Its Shock Absorber System
}

\author{
Dimitrios Giagopoulos ${ }^{1, *(1)}$, Alexandros Arailopoulos ${ }^{1}(\mathbb{C})$ and Iraklis Chatziparasidis ${ }^{1,2}$ (D) \\ 1 Department of Mechanical Engineering, University of Western Macedonia, 50100 Kozani, Greece; \\ aarailopoulos@uowm.gr (A.A.); i.chatziparasidis@kleemannlifts.com (I.C.) \\ 2 Engineering Research Department, Kleemann Hellas SA, 61100 Kilkis, Greece \\ * Correspondence: dgiagopoulos@uowm.gr; Tel.: +30-2461-56751
}

Citation: Giagopoulos, D.;

Arailopoulos, A.; Chatziparasidis, I. Optimal Modeling of an Elevator

Chassis under Crash Scenario Based on Characterization and Validation of the Hyperelastic Material of Its Shock Absorber System. Appl. Mech. 2022, 3 , 227-243. https://doi.org/10.3390/ applmech3010016

Received: 31 January 2022

Accepted: 3 March 2022

Published: 5 March 2022

Publisher's Note: MDPI stays neutral with regard to jurisdictional claims in published maps and institutional affiliations.

Copyright: () 2022 by the authors. Licensee MDPI, Basel, Switzerland. This article is an open access article distributed under the terms and conditions of the Creative Commons Attribution (CC BY) license (https:// creativecommons.org/licenses/by/ $4.0 /)$.

\begin{abstract}
A wide variety of hyperelastic rubber-like materials, exhibiting strong nonlinear stressstrain relations under large deformations, is applied in various industrial mechanical systems and engineering applications involving shock and vibration absorbers. An optimal design procedure of an elevator chassis crashing on a hyperelastic shock absorber in a fail scenario, applicable in large-scale mechanical systems or industrial structures of high importance under strong nonlinear dynamic excitation, is presented in this work. For the characterization of the hyperelastic absorber, a Mooney-Rivlin material model was adopted, and a series of in-lab compression quasi-static tests were conducted. Applying a fully parallelizable state-of-the-art stochastic model updating methodology, coupled with robust, accurate and efficient Finite Element Analysis (FEA) software, the hyperelastic behavior of the shock absorber was validated under uniaxial large deformation, in order to tune all material parameters and develop a high-fidelity FE model of the shock absorber system. Next, a series of in situ full-scale experimental trials were carried out using a test-case elevator chassis, representing the crash scenario on the buffer absorber system, after a controlled free fall. A limited number of sensors, i.e., triaxial accelerometers and strain gauges, were placed at characteristic points of the real structure of the elevator chassis recording experimental data. A discrete Finite Element (FE) model of the experimentally tested arrangement involving the elevator chassis and updated buffer absorber system along with all boundary conditions was developed and used in explicit nonlinear analysis of the crash scenario. Steel material properties and the characterized updated Mooney-Rivlin material model were assigned to the elevator chassis and buffer, respectively. A direct comparison of the numerical and experimental data validated the reliability and accuracy of the methodology applied, whereas results of the analysis were used in order to redesign and optimize a new-design elevator chassis, achieving minimum design stresses and satisfying serviceability limit states.
\end{abstract}

Keywords: nonlinear crash analysis; FE model updating; material nonlinearities; hyperelastic material

\section{Introduction}

The advancement of technology in new rubber-like elastomer materials, exhibiting strong nonlinear stress-strain relations under large deformations, has increased in recent years and gained the attention of researchers in the area of continuum mechanics, leading to the development of products and applications implemented in various industrial mechanical systems and engineering problems involving shock and vibration absorbers. An elastomer is a type of material that can elastically recover to its initial state and shape after deforming from tension or compression. Elastomers that exhibit large reversible strains in response to a compression or tension stress belong to the family of polymers [1-3]. Elastomers, being theoretically perfect polymers, along with great abrasion and chemical resistance as well as air and water impermeability, present viscoelastic properties and great resistance under static or dynamic stresses, under various climatic conditions and 
temperature range. These properties of elastomers render its use suitable for damping mechanisms such as vibration and shock absorption applications [2,4-6].

The property characteristics of elastomers highly depend on the composition of the compound, characterized by the type and amount of rubber chemicals and additives used, as well as its chemical and mechanical treatment during production, which influences hardness, elasticity and strength. Thus, elastomer compound material properties are inherently sensitive to the rubber selection, the composition of the compound and the manufacturing process as well as the final geometrical shape and design of the final product. Moreover, the type of loading affects the elastomer properties, presenting different behavior under static, quasi-static or dynamic excitation. Due to its material variability and broad implementation, elastomer applications and products strongly require certification results through numerical or combined numerical and experimental validation [7-9].

Thus, it is of high importance to fully understand and increase confidence about the mechanical behavior and loading capacity of each elastomer product. Moreover, as most engineering applications require multi-axial loading strength, their behavior in specific loading conditions also needs to be examined. Combined experimental measurements, conducted in and out of a laboratory, and numerical Finite Element (FE) model simulations are employed in order to investigate the macroscopic mechanical characteristics and material properties of an elastomer application $[9,10]$.

In this work, an optimal design procedure of an elevator chassis crashing on a highly elastic cellular polyurethane elastomer shock absorber in a fail scenario is presented. At first, specimens of the real rubber-like absorber were tested under uniaxial compression deformations in a loading machine, extracting experimental force-displacement curves. A discrete (FE) model of the examined absorber was developed and a compressible MooneyRivlin material model was assigned. The material properties of the specific polyurethane elastomer shock absorber structure are classified and tuned reconciling experimental data to equivalent numerical (FE) model computations. This is achieved using residuals based on the experimental and numerical time histories of displacements [11,12].

Structural model parameter updates, based on an experimentally measured model and response data, are usually formulated as weighted least-squares estimation residuals. Although prone to local optima entrapment, conventional gradient-based optimization techniques are then used to find the optimal values that minimize the single-objective residual. Alternatively, evolutionary strategies (ES) [13] are more effective in guaranteeing convergence to a global optimum, coming short of convergence rates. In this work, a non-gradient state-of-the-art optimization algorithm, i.e., Covariance Matrix AdaptationEvolution Strategy (CMA-ES) [14-16], coupled to a commercial FEA solver is applied in a parallel computing scheme to solve the single-objective optimization problem, updating model parameters without the need for model reduction or sub-structuring techniques [17].

Using the updated material properties and characterizing the nonlinear hyperelastic behavior of the elastomer absorber, an explicit nonlinear analysis of a test-case elevator system crashing on the buffer absorber was carried out followed by a series of full-scale in situ experimental trials, representing the crash scenario of the elevator chassis on the buffer absorber system after a controlled free fall. The hyperelastic behavior of the shock absorber was validated under uniaxial large deformation, and a new-design elevator chassis was redesigned and optimized in order to achieve minimum design stresses and deformations.

The novelty of the presented procedure in its wholeness includes both computational and engineering novel aspects. From a computational point of view, contribution is attributed to the integration of a state-of-the-art optimization algorithm, CMA-ES, applied in parallel computing and coupled to a robust (FE) model analysis solver in order to produce an optimal design for a real industrial engineering problem. Aiming at a reliable simulation of a fail scenario of a flexible elevator chassis crashing on a highly elastic cellular polyurethane elastomer shock absorber of unknown mechanical and material behavior, characterization and validation of its material model and parameters needed to be addressed accurately and effectively. Thus, novel computational aspects pertain to the 
applicability of the CMA-ES optimization algorithm in order to finely tune the unknown material properties, resulting in the characterization of its mechanical behavior through a FEM update procedure devoid of model reduction or data expansion techniques. From an engineering point of view, the applied procedure in total is the major contribution in applications of engineering science. Combining results from an in-lab experimental arrangement involving the unknown part, along with the implementation of the above computational tool, the mechanical characteristics and material properties of the applied elastomer were tuned and validated, developing a high-fidelity FEM model of the shock absorber. Furthermore, the elastomer behavior was adequately validated from the subsequent in situ experimental trials of a full-scale high-rigidity test-case elevator system falling freely on the examined absorber. The validated results concluded to confidently introduce the FEM of the shock absorber using the characterized material model and parameters in order to fulfill the main engineering target of the optimal design of the final industrial product of a flexible elevator chassis for further commercial trade.

The presentation in this work is organized as follows. The theoretical formulation of the Finite Element model updated based on time histories of displacements is briefly presented in Section 2, whereas Section 3 summarizes the applied FE model updating framework. Section 4 presents the in-lab experimental application and the development of the equivalent FE model of a polyurethane elastomer shock absorber under quasi-static excitation in a tension-compression machine. Section 5 presents the FE model updating parameterization and the validation results of the hyperelastic material characterization. Next, Section 6 presents the in situ validation of the updated characterized hyperelastic material using a test-case elevator chassis under a crash scenario. Finally, in Section 7 the updated and in situ validated hyperelastic material properties are utilized for the optimal design and verification of a full-scale new-design elevator system crashing on the buffer absorber. Conclusions are summarized in the final Section 8.

\section{Finite Element Model Updating Formulation based on Displacement Response Time Histories}

Let $\mathrm{M}$ be a parameterized class of structural dynamic models, which simulates the structural system and will be used to estimate desired quantities of interest. Consider $\underline{\theta}_{\mathrm{m}} \in \mathrm{R}^{\mathrm{N}_{\theta}}$ the set of free structural model parameters to be adjusted using the measured data and let $g\left(\underline{\theta}_{m} \mid M_{m}\right)$ be the model predictions given the values of the parameter set $\underline{\theta}_{m}$. In this work, parameter estimation is based on response time history measurements of displacements. This formulation has the advantage of applicability over both linear and non-linear systems; it compares the measured raw data of the experimental arrangement to the equivalent predictions of the numerical model. In this way, all available information is preserved, and systematic errors of the identification procedure are alleviated.

The difference between the measured response time histories and the model predicted response time histories take the following form [18]:

$$
\mathrm{J}(\underline{\theta} ; \mathrm{M})=\frac{1}{\mathrm{~m}} \sum_{\mathrm{i}=1}^{\mathrm{n}} \frac{\sum_{\mathrm{j}=1}^{\mathrm{m}}\left(\underline{\mathrm{g}}_{\mathrm{ij}}\left(\underline{\theta}_{\mathrm{m}} \mid \mathrm{M}\right)-\hat{\mathrm{y}}_{\mathrm{ij}}\right)^{2}}{\sum_{\mathrm{j}=1}^{\mathrm{m}}\left(\hat{\mathrm{y}}_{\mathrm{ij}}\right)^{2}}
$$

where $\underline{g}_{i j}\left(\underline{\theta}_{m} \mid M\right)$ is the numerical time history of the introduced FE model and $\hat{y}_{\mathrm{ij}}$ is the respective experimental signal. Subscript i corresponds to the sensor location and measurement direction, and $\mathrm{j}$ corresponds to the time-step instant. $\mathrm{n}$ is the total number of measured sensor locations and directions, whereas $\mathrm{m}$ is the total number of measured time-steps (number of observations).

The measure of fit introduced in Equation (1) consists of displacements equally weighted, expanding to the following Equation (2), where $\mathrm{d}\left(\underline{\theta}_{\mathrm{m}}\right)_{\mathrm{ij}}$ is the numerical time histories of displacements of the introduced FE model and $\widehat{\mathrm{d}}_{\mathrm{ij}}$ is the respective experimental displacement signal. Additionally, responses from all locations and components are 
included in the produced measure of fit in order to maximize the fidelity of the updated model resulting in Equation (2).

$$
\mathrm{J}(\underline{\theta})=\frac{1}{\mathrm{~m}} \sum_{\mathrm{i}=1}^{\mathrm{n}} \frac{\sum_{\mathrm{j}=1}^{\mathrm{m}}\left(\mathrm{d}\left(\underline{\theta}_{\mathrm{m}}\right)_{\mathrm{ij}}-\widehat{\mathrm{d}}_{\mathrm{ij}}\right)^{2}}{\sum_{\mathrm{j}=1}^{\mathrm{m}}\left(\widehat{\mathrm{d}}_{\mathrm{ij}}\right)^{2}}
$$

It is worthwhile to mention that during the experimental measurements, the loading under which the buffer is excited is recorded, and the displacement responses and the measured force is introduced as excitation in the FE model.

\section{Applied FE Model Updating Framework}

The examined deterministic model is not a simple analytical model where the dependence on the parameters is explicit. Thus, a stochastic black-box search algorithm, where provided inputs produce equivalent outputs, needs to be used. Covariance Matrix Adaptation Evolution Strategy (CMA-ES) $[15,16]$ is used to this end due to the following advantages. CMA-ES is a randomized black box search algorithm that uses multivariate normal distributions to sample new search points. The covariance matrix of the distribution is not restricted a priori, in contrast to many continuous domain evolutionary algorithms that use a single normal search distribution. Compared to computationally faster gradientfree local optimizers such as Nelder-Mead simplex direct search algorithm available in MATLAB fminsearch function, CMA-ES is suitable in finding the global optimum, avoiding being trapped at local optima, and has demonstrated rapid convergence capabilities, particularly when searching for a global optimum compared to other evolution algorithms. Furthermore, it is a general purpose method which has been applied successfully to linear and non-linear FE updating problems $[19,20]$, involving large and complex models and cases. Last but not least, it is fully parallelizable, compensating for computational time to convergence when compared to other sequential iterative gradient-free techniques such as the MATLAB fminsearch algorithm.

The computational framework applied in this work [17] involves an iterative process, during which the solution is being sought by randomly sampling a search space. Sampling the best weighting residuals found in each set of iterations (function evaluation) to update the initial chosen statistical values (mean, covariance matrix), the global minimum of the applied objective function is iteratively approached.

As CMA-ES is fully parallelizable, in this work a C programming language free distribution of the CMA-ES algorithm is applied in parallel computing to solve the singleobjective optimization problem, arising from the deterministic model formulation. Robust and accurate FE Analysis software DYNAMIS [21] is employed, in combination with the parallelized strategy, in order to produce results of the proposed objective function residual simultaneously and populate the algorithm's o springs.

The following diagram in Figure 1 presents the flow of a single iteration (complete set of function evaluations), beginning from the collection of experimental time history data, including both response time histories and measured excitations. Next, a Multivariate Normal Search Distribution, having the largest entropy of all distributions in $\Re^{\mathrm{n}}$ given all variances and covariances, is employed in order to produce a new parameter set $\underline{\theta}$. FE Analysis software coupled to CMA Evolution Strategy is invoked in order to compute the equivalent numerical (predicted) time domain displacement $d(\underline{\theta})$ responses. Next, the selected deterministic objective function is calculated and a single offspring of the chosen population $\lambda$ is produced.

The described framework runs in parallel, i.e., it takes advantage of the HyperThreading Technology of the working CPUs, in order to sample the population $\lambda$ simultaneously so as to complete a total iteration (set of function evaluation). The CMA-ES algorithm, upon collecting the prescribed amount of offsprings calculated in the above function evaluation, checks if convergence criteria are met. Various criteria were intro- 
duced in order to take control and affect the speed of the optimization process. Firstly, a given threshold of the objective function $\mathrm{J}(\underline{\theta} ; \underline{\mathrm{w}})=0$ is used. This criterion is practically inapplicable, as in experimental cases, with many uncertainties and model errors, the minimum is unknown beforehand and $\mathrm{J}(\underline{\theta} ; \underline{\mathrm{w}}) \neq 0$. Alternatively, two similar criteria, regarding standard deviations and the difference of the best values of two consecutive function evaluations $\Delta \mathrm{J}(\underline{\theta} ; \underline{\mathrm{w}})=10^{-3}$ are used. Moreover, in order to prevent the process of running indefinitely, a given limit of the number of iterations is also being introduced, namely $\mathrm{N}_{\mathrm{s}}=28,800$. If none of the convergence criteria are met, the process recommences.

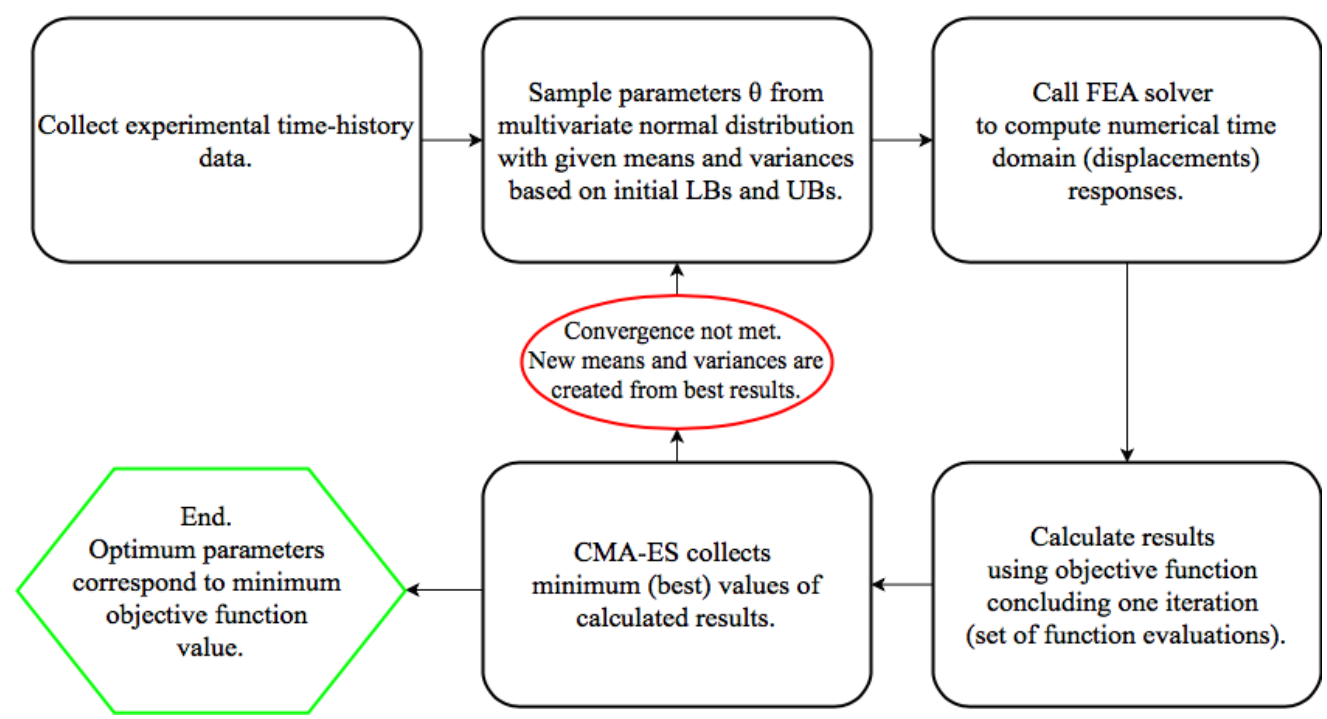

Figure 1. Flow diagram of the applied CMA-ES framework.

The proposed framework surpasses model reduction or data expansion techniques. Due to the significantly large number of system runs required, the computationally expensive simulations of CMA-ES are managed by a parallelized version using the non-intrusive adaptive Parallel Numerical Differentiation Library (PNDL) [22]. The framework is implemented within $\Pi 4 \mathrm{U}$ framework [13] based on a state-of-the-art task-parallel library for clusters, called TORC [23], which is designed to provide unified programming and runtime support for computing platforms that range from single-core systems to hybrid multicore-GPU clusters and heterogeneous grid-based supercomputers.

A C programming language version of the CMA-ES algorithm, and FE Analysis software DYNAMIS [21,24], were coupled through MATLAB [25], having the framework compiled and run independently in parallelization. The computer that was used hosts two (2) Intel ${ }^{\circledR}$ Xeon ${ }^{\circledR}$ Processors E5-2630 v3 (20 M Cache, 2.40 GHz) with 8-cores and 16-threads, resulting in a total number of thirty-two (32) logical (virtual) cores and 64 GB of RAM on a Linux Ubuntu 16.04 Operating System.

\section{Compression Test for Hyperelastic Material Characterization}

\subsection{Compressible Mooney-Rivlin Rubber-Like Material Behavior}

In this work, the rubber-like material was modeled introducing the commonly used Mooney-Rivlin material model $[6,26,27]$ in order to define the strain energy density function $\bar{W}$ per unit of original volume. The conventional incompressible Mooney-Rivlin material model is described by the following Equation (3):

$$
\overline{\mathrm{W}}=\mathrm{A}_{10}\left(\mathrm{I}_{1}-3\right)+\mathrm{A}_{01}\left(\mathrm{I}_{2}-3\right) ; \mathrm{I}_{3}=1
$$

where $\mathrm{A}_{10}$ and $\mathrm{A}_{01}$ are material constants determined empirically and $\mathrm{I}_{\mathrm{i}}, \mathrm{i}=1,2,3$ are the strain invariants: 


$$
\begin{aligned}
& \mathrm{I}_{1}=\lambda_{1}{ }^{2}+\lambda_{2}{ }^{2}+\lambda_{3}{ }^{2} \\
& \mathrm{I}_{2}=\lambda_{2}{ }^{2} \lambda_{3}{ }^{2}+\lambda_{3}{ }^{2} \lambda_{1}{ }^{2}+\lambda_{1}{ }^{2} \lambda_{2}{ }^{2} \\
& \mathrm{I}_{3}=\lambda_{1}{ }^{2} \lambda_{2}{ }^{2} \lambda_{3}{ }^{2}
\end{aligned}
$$

where $\lambda_{1}, \lambda_{2}, \lambda_{3}$ are the cuboid dimensions of a deformed cube of the material with unit edges.

As the above description accepts $I_{3}=1$, the bulk modulus is regarded several thousand times larger than the shear modulus and a totally incompressible material model is assumed. For a compressible Mooney-Rivlin rubber the strain energy density function is defined by the following Equation (5):

$$
\overline{\mathrm{W}}=\mathrm{A}_{10}\left(\mathrm{I}_{1}-3\right)+\mathrm{A}_{01}\left(\mathrm{I}_{2}-3\right)+\mathrm{C}\left(\frac{1}{\mathrm{I}_{3}^{2}}-1\right)+\mathrm{D}_{1}\left(\mathrm{I}_{3}-1\right)^{2}
$$

where

$$
\begin{aligned}
& \mathrm{C}=\frac{1}{2} \mathrm{~A}_{10}+\mathrm{A}_{01} \\
& \mathrm{D}_{1}=\frac{\mathrm{A}_{10}(5 v-2)+\mathrm{A}_{01}(11 v-5)}{2(1-2 v)}
\end{aligned}
$$

Additionally, $v$ is the Poisson's ratio laying between 0.49 and 0.5 .

\subsection{Experimental Arrangement}

In order to examine the complexity and material mechanical behavior of the used polyurethane elastomer shock absorber, a quasi-static compression experimental test was conducted. The compression experiment as a fundamental type of mechanical test of a material was used for the quantitative representation of the change in force as a function of deformation, from which a first estimate of the mechanical response of the hyperelastic material can be deducted. Additionally, the compression test allowed the quantification of the limit loads at which changes in response of the hyperelastic material are observed, such as transition from elastic to inelastic behavior. Finally, during the compression test the chosen hyperelastic material model is evaluated along with the effect of its parameters or its structural complexity as it appears in multiple geometric scales. Figure 2 (left) presents the experimental set-up of the buffer during the compression test. Specifically, this figure shows the tension-compression experimental machine and its controller board along with its load cell and linear position sensors. The top and bottom plates are made of steel with a thickness of $10 \mathrm{~mm}$ each. The examined polyurethane elastomer shock absorber is placed between them, being compressed only from the top plate, which is constrained in vertical direction, whereas the bottom plate remains fixed. Both plates do not rotate in all three vertical planes. During the experiment, the applied force is equally distributed on the whole surface of the buffer avoiding local concentration of the compression force, that could result in abnormal distortion and stress concentration in the area of applied force, clearly violating the Saint-Venant principle.

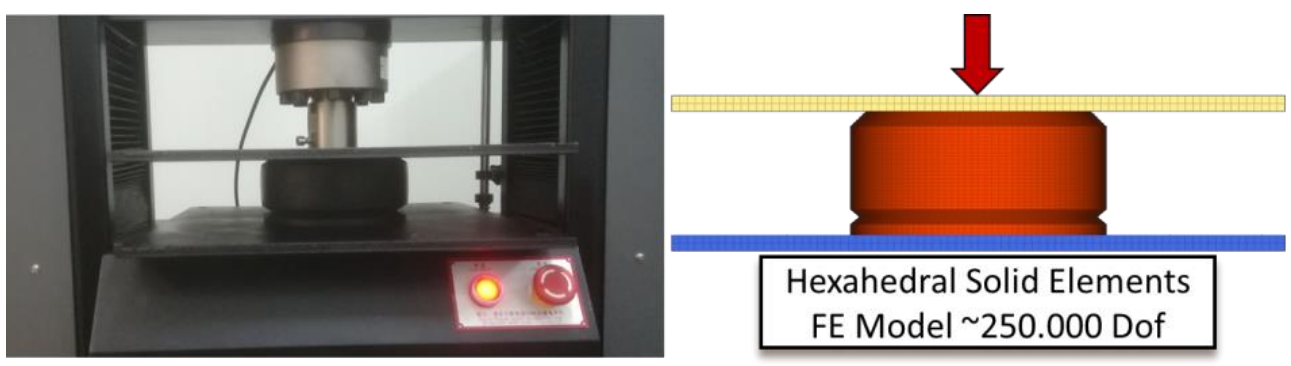

Figure 2. Experimental set-up of the buffer in the tension-compression test machine (left) and FE model of buffer and steel plates equivalent to the experimental set-up (right).

Moreover, the plates used to obtain a uniform distribution of stresses allow its free lateral expansion. This is achieved by reproducing the actual way the buffer is mounted on 
the elevator chassis. Thus, the buffer is only clamped on the bottom plate in the middle using a steel ring, which is actually part of the buffer system. The rest of the buffer is not rigidly attached to the steel plates, being only in contact, smooth enough not to impede lateral expansion. While such end conditions could be achieved for the buffer in compression, they cannot be physically realized in the case of tension, but such a test is not needed, as the buffer system is only loaded in compressive forces and does not operate in tension conditions.

The test was performed at a low compression rate so that it responds to quasi-static loading. Specifically, a displacement-controlled procedure was selected using a displacement rate of $2 \mathrm{~mm} / \mathrm{min}$ or $1 / 3 \mathrm{~mm} / \mathrm{s}$. During the test, the machine continuously recorded force, displacement and time. The phenomenon known as the Mullins effect [28,29], where the softening of the stress-strain curve of the examined rubber-like material depends on the sequence and level of each loading cycle, is not considered. Such a discontinuous damage model involving a progressive irreversible loss of stiffness of the rubber absorber after a few maximum strain level occurrences is not taken into account, both for the in-lab and for the following in situ experiments. The reason is simply that the shock absorbers were brand new, used only once in the experiment conducted in the lab and were discarded after each trial.

The equivalent Finite Element model of the presented laboratory experimental compression test set-up is presented in Figure 2 (right). Both the geometry of the buffer and the steel plates are discretized with tetrahedral solid elements using appropriate pre-processing commercial software [30]. The total number of DOFs of the final model, representing the experimental setup is approximately 250,000 [31]. Contact analysis was carried out meaning that the examined bodies were regarded as deformable and interacting with each other. A toughing contact type was selected, where normal forces act on the contacting surfaces of the adjacent bodies when they touch each other, and additional shear forces are also developed due to friction between the touching surfaces at a specified friction coefficient of 0.25 provided by the manufacturer of the shock absorber against steel plate. Bodies are allowed to slide against each other and are allowed to move according to the applied boundary conditions and constraints of the model, without penetrating one another. Contact analysis was introduced in all subsequent analyses in order to accurately simulate the deformation of the shock absorber that is vertically compressed and laterally elongated by sliding against the surrounding steel using a friction coefficient provided by the manufacturer.

The nominal material parameters of steel parts have been chosen as Young's modulus $\mathrm{E}=210 \mathrm{GPa}$, Poisson's ratio $v=0.3$ and density $\rho=7850 \mathrm{~kg} / \mathrm{m}^{3}$. The material properties of the polyurethane elastomer shock absorber correspond to a fully nonlinear (large strain and large rotation) hyperelastic rubber-like material of a compressible Mooney-Rivlin model $[6,26,27]$ with parameters $\mathrm{A}_{10}=1.2, \mathrm{~A}_{01}=0.001, \mathrm{D}_{1}=5000, v=0.49997$ and $\rho=400 \mathrm{~kg} / \mathrm{m}^{3}$. Alternatively, although the Blatz-Ko hyperelastic material model [32,33] could be proven adequate, it was not examined due to limitations in modeling the mechanical behavior of materials with a large Poisson's ratio, its inability to predict the whole range of strain responses as well as its limited parameterization potential (one input parameter) within the accommodated solver DYNAMIS. Additionally, there is a steel ring holding the buffer to the bottom plate which has the nominal steel material parameters. The measured time history of applied force is introduced in the Finite Element model as excitation force in a transient analysis in order to finely tune the Mooney-Rivlin material parameters.

\section{FE model Update and Validation of Compression Test Results}

\subsection{Parameterization of FE Model}

The parameterization of the Finite Element model is introduced in order to facilitate the applicability of the updating framework. The parameterized model consists of four (4) parts, as shown in Figure 3. The diameter of the buffer is $167 \mathrm{~mm}$ with a height of $81 \mathrm{~mm}$, while the plates are square with a side of $400 \mathrm{~mm}$ and a thickness of $10 \mathrm{~mm}$. Parts 
1-3 are modeled with solid elements and isotropic material properties, complying to the generalized Hooke's Law of elasticity due to small strain development, whereas part 4 is modeled with solid elements and hyperelastic Mooney-Rivlin material properties. All material properties are used as design variables in the model updating process, resulting in ten (10) design variables, pertaining to all moduli of elasticity $\mathrm{E}$ and densities $\rho$. The $\mathrm{D}_{1}$ parameter of the Mooney-Rivlin material model is dependent on $\mathrm{A}_{10}, \mathrm{~A}_{01}$ and $v$ parameters, whereas Poisson's ratio ranges between $v=[0.49,0.50]$. Additionally, ten (10) decimals were regarded in the FEM updated process due to the high sensitivity of $\mathrm{D}_{1}$ to changes in $v$. Thus, in this work, a procedure where a nonlinear Poisson's function could be characterized at first, was not examined for the adopted material model and the confidence in its implementation will build on the validation of the in-lab and in situ experimental data to simulation predictions for small and large strain and displacement conditions.
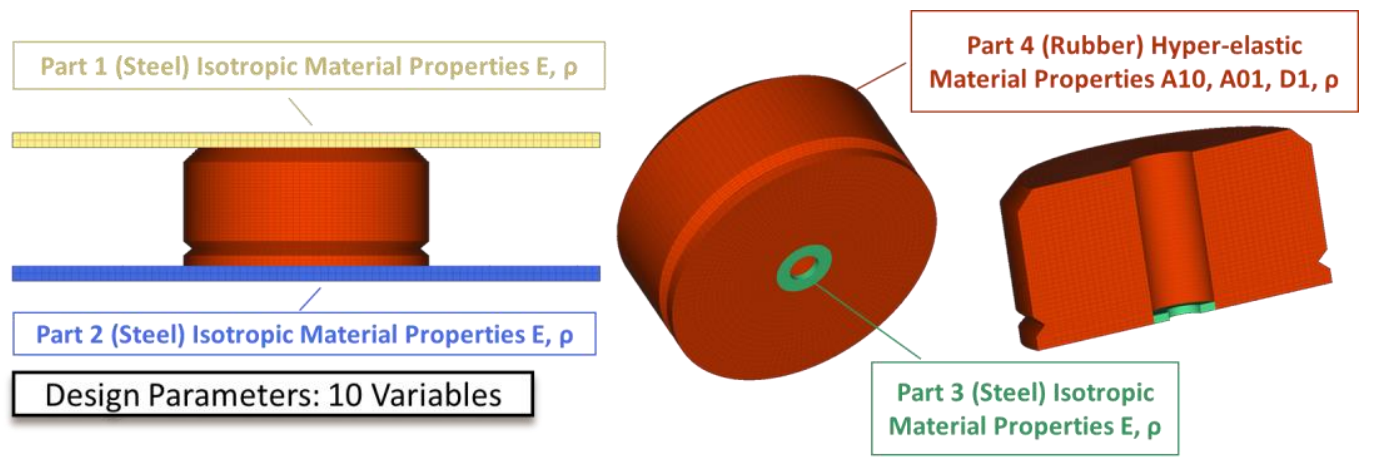

Figure 3. Parts of the parameterized FE model.

Applying the measured load excitation to the FE model, the displacements were numerically computed. The CMA-ES framework is applied using a range of $\pm 10 \%$ from the nominal values for the design bounds, in order to update the developed FE using the objective function presented in Equation (2), which quantifies the discrepancy between experimentally measured and numerically predicted displacement time domain responses. The set of parameter values presented in the previous Section 4.1 was chosen as the initial statistical values (means) in the CMA-ES algorithm as described in Section 3. These means along with variances computed from the above range of variation will commence the initial iteration including the first set of function evaluations from which the minimum values with be selected in order to proceed to the next iteration until convergence criteria are met. The optimization process performed forty (40) iterations concluding one hundred (100) function evaluations per iteration, at approximately two (2) minutes per run. Thus, the total number of iterations was approximately 4000 completed in approximately four (4) hours, due to the thirty-two (32) parallelized-scheme of the applied CMA-ES methodology.

\subsection{Validation of Results}

After convergence of the CMA-ES framework, the updated parameters are introduced in the FE model and the predicted responses are graphically compared to the experimental measurements. Figure 4a presents a comparison between measured experimental time histories of displacement against the numerically predicted respective ones of the updated FE model. The red continuous line represents the experimentally measured response, and the green continuous line presents the predicted response.

Similarly, Figure $4 \mathrm{~b}$ presents the measured imposed load against the experimental and numerically computed displacements. The same colors apply as before.

The presented result increased confidence of a high-fidelity FE model characterizing the examined polyurethane elastomer shock absorber material and is next appropriately used in the subsequent full-scale elevator chassis under a fail-safe scenario. 

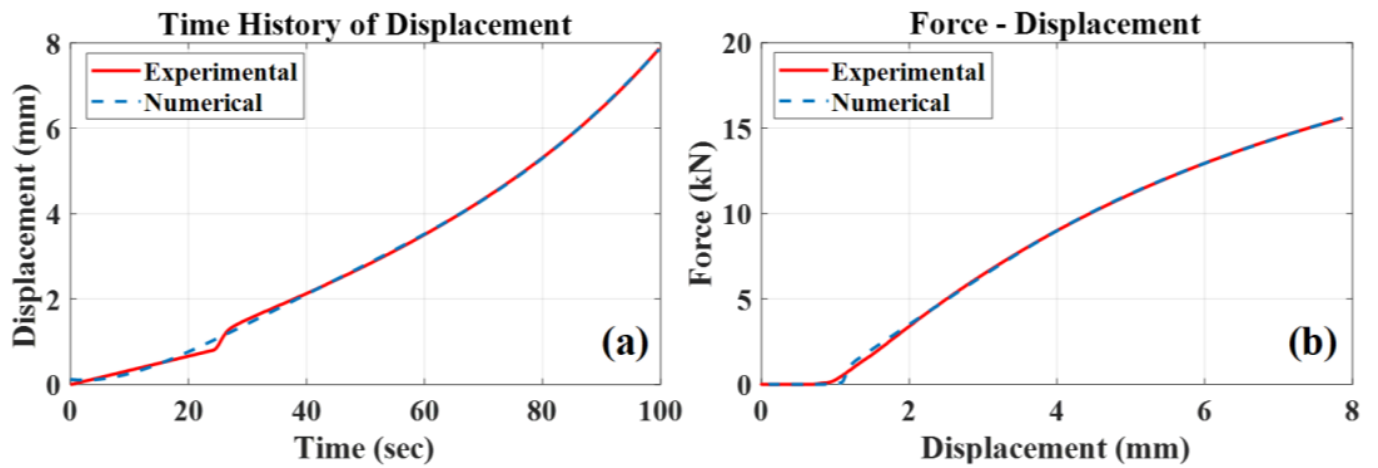

Figure 4. (a) Comparison of experimental and numerical of updated FEM time histories of displacement and (b) imposed force-displacement comparison between experiment and updated FEM analysis.

The following Figure 5 indicatively presents the initial and final state of the shock absorber, from the experiment and the analysis of the updated FE model, colored by contours of displacements.
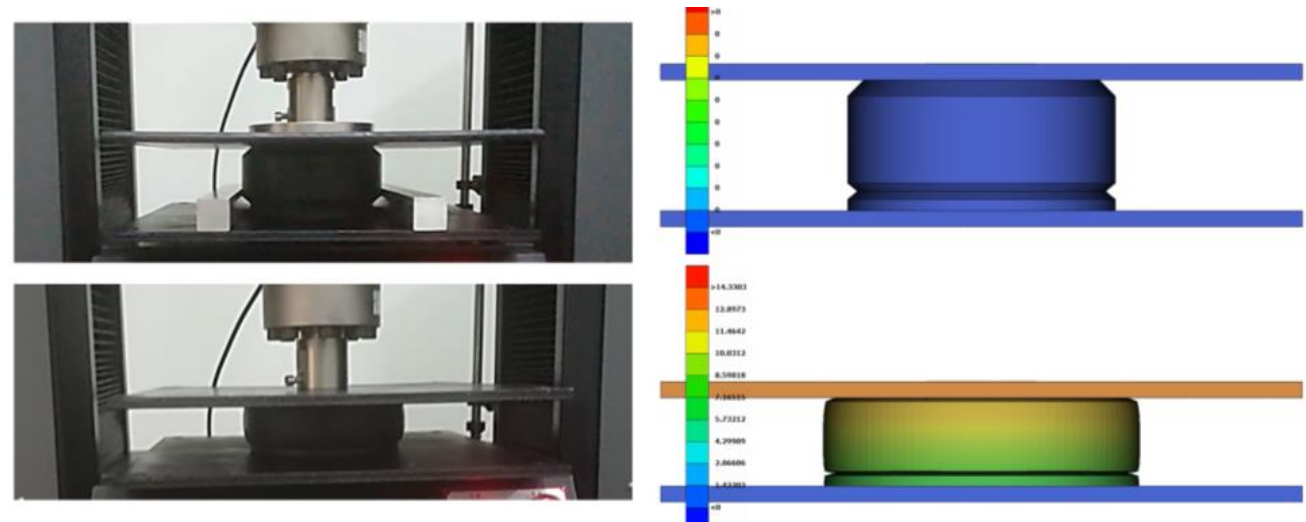

Figure 5. Comparison between experimental and numerical response at final states of compression.

\section{In Situ Validation of Characterized Hyperelastic Material Model}

The complete elevator system (test-case chassis), used in the in situ experiment is shown in Figure 6 with the corresponding Finite Element model. The geometry of the chassis was discretized using mainly rectangular and triangular shell finite elements. Additionally, solid (hexahedral) elements, rigid body elements and some other special elements are used. The total number of degrees of freedom in the resulting FE model is about 2,500,000.

In order to test the reliability of the applied FE model update methodology, four (4) strain gauges (HBM) and five (5) $\pm 50 \mathrm{~g}$ triaxial accelerometers (BK 4535-B-001 type with frequency range of $0.3-10,000 \mathrm{~Hz}$ ) are placed at selected positions of the chassis and a set of measurements is carried out under real dynamic loading conditions-a crash scenario with impact velocity of the chassis to the absorber $1.3278 \mathrm{~m} / \mathrm{s}$, using a data acquisition system of National Instruments (cDAQ 9178, with modules 9234 and 9237). The sensor locations are presented in Figure 7 and include the strain gauge locations (SG1, SG2, SG3 and SG4) and the accelerometer locations (A1, A2, A3, A4 and A5). For a complete monitoring of the stress state, three bridges with a $90 \mathrm{o}$ angle rosette were placed at each of these locations. At each measurement location, the stresses calculated are the normal stresses $\sigma_{\mathrm{x}}$ and $\sigma_{\mathrm{y}}$, the shear stress $\tau_{x y}$ and the maximum equivalent von Mises stress. 

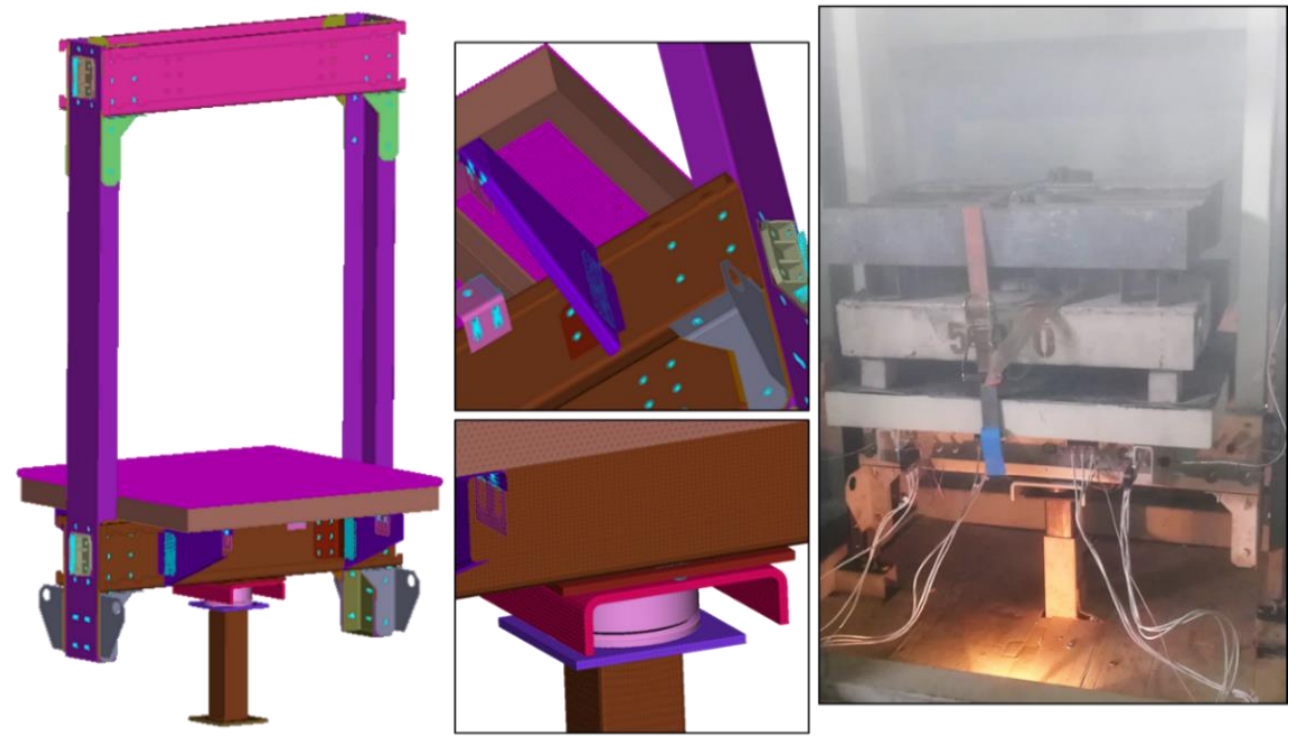

Figure 6. Elevator System, (left) Finite Element Model and (right) Experimental set-up.

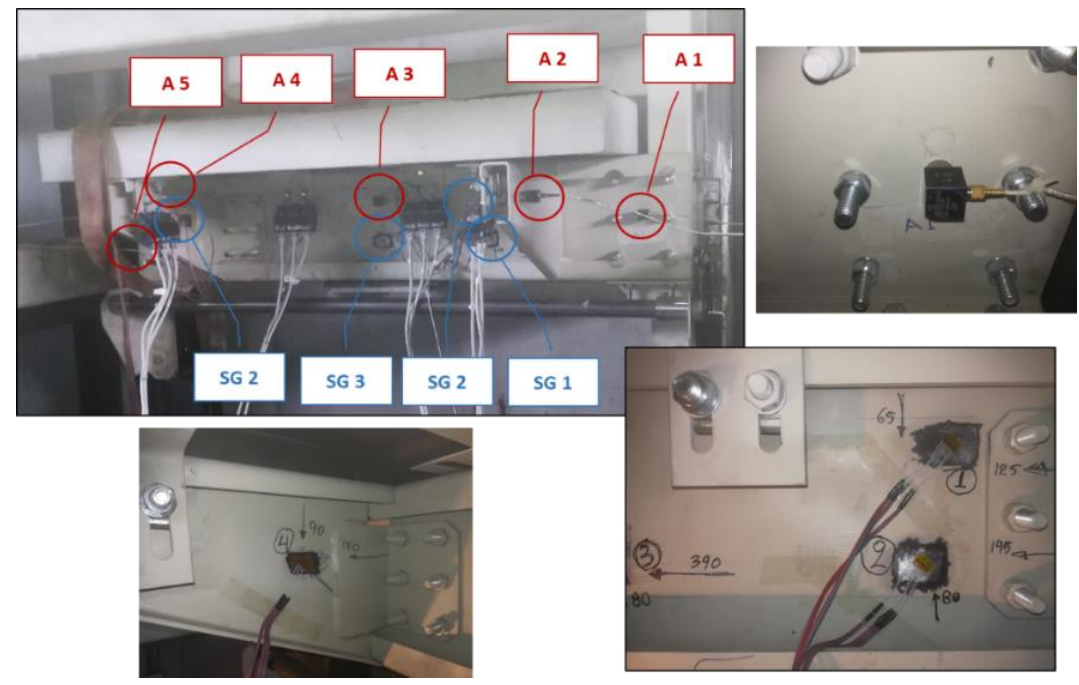

Figure 7. Strain gauges and accelerometers located on the in situ test-case chassis.

For the equivalent FE simulation, an explicit nonlinear response analysis was performed using nominal material properties for the steel chassis of the elevator components where Young's modulus is $\mathrm{E}=210 \mathrm{GPa}$, Poisson's ratio $v=0.3$ and density $\rho=7850 \mathrm{~kg} / \mathrm{m}^{3}$. An elastoplastic, elastic-full plastic material behavior was selected for the steel material, having a yield stress at $\sigma_{\text {yield }}=355 \mathrm{MPa}$, meaning that the behavior of the steel is perfectly elastic, up to the yield stress limit. If yield stress is reached, deformation is progressing with no further stress development on the material. Respectively, the updated parameters of the hyperelastic Mooney-Rivlin material model were assigned to the polyurethane elastomer shock absorber. An explicit nonlinear transient response analysis was carried out within DYNAMIS at a time step of $\Delta \mathrm{t}=10^{-7} \mathrm{~s}$. The time-step size chosen for stability of the analysis was selected based on the Courant-Friedrichs-Lewy (CFL) condition, considering the smallest element characteristic length and an approximation for the longitudinal wave speed. It is worth mentioning that although the elastomer absorber is deformed at maximum levels, the Mullins effect [28,29] does not apply to this specific arrangement. The reason is that the shock absorber part is rendered useless and replaced after each crash test.

Figure 8 presents the results of the displacements from the numerical analysis of the Finite Element model. Specifically, an instant in time of the test-case elevator chassis 
colored by contours of displacements is presented in the right part of Figure 8, whereas the right part compares the deformation of the buffer at the instant of maximum displacement between the numerical analysis results and the experimentally measured data from a highspeed camera. It is worth mentioning that the strain-rate effect is insignificant for this test and therefore not examined.
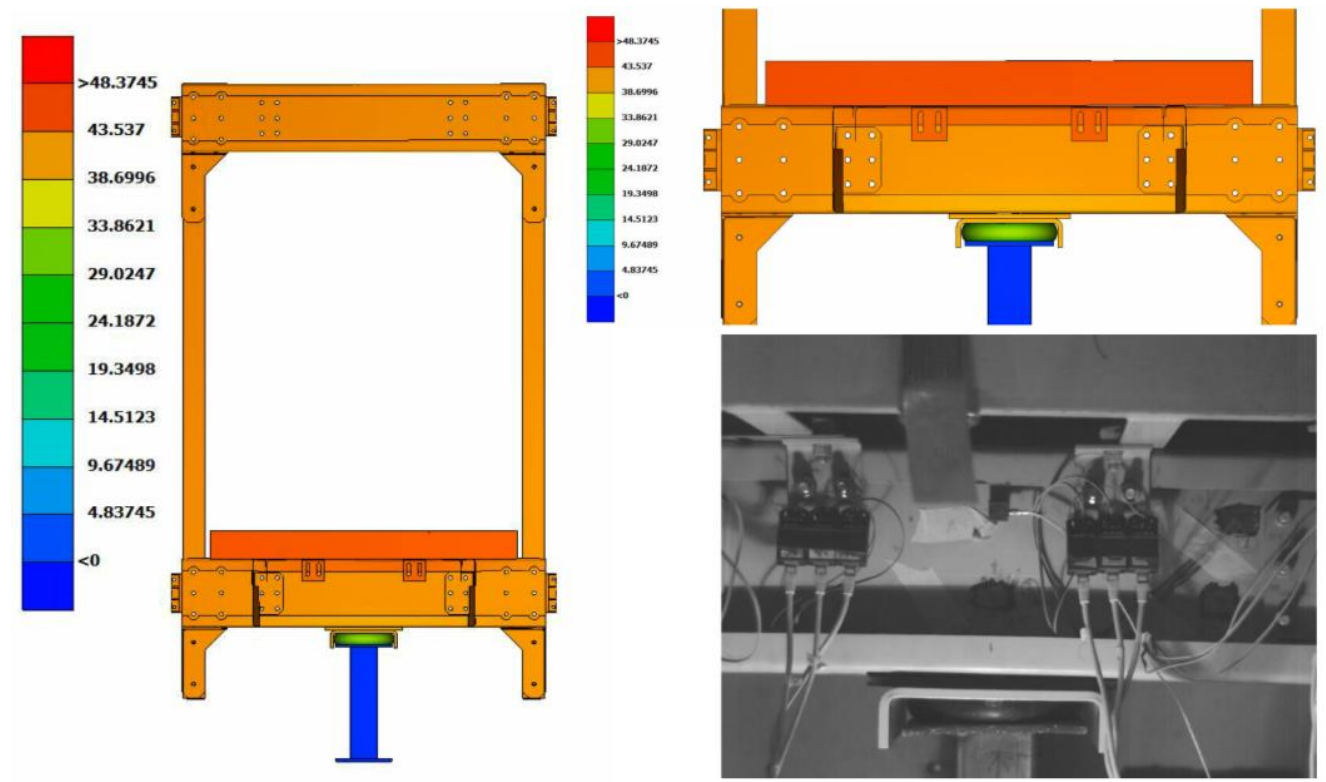

Figure 8. Numerical analysis results and comparison of total deformation $(\mathrm{mm})$ between numerically predicted and experimentally measured maximum displacement.

Moreover, Figure 9 details the comparison between the numerical results and the experimental measurements, for the lateral and vertical deformation of the buffer at the maximum displacement position. The experimental results were obtained from high-speed camera images. Specifically, at maximum displacement the lateral deformation of the buffer results in a total diameter of $203.20 \mathrm{~mm}$ from the high-speed camera images, compared to a total diameter of $205.20 \mathrm{~mm}$ predicted by the numerical analysis, validating the characterization of the hyperelastic material model of the shock absorber and its behavior in the lateral direction. Additionally, at maximum displacement the vertical deformation of the buffer resulted in $33.42 \mathrm{~mm}$ from the high-speed camera images, compared to approximately $34.36 \mathrm{~mm}$ predicted by the numerical analysis, validating also the shock absorber behavior in the vertical direction.

Figure 10 shows the distribution of equivalent von-Mises stresses on the test-case chassis at various instants of time where the red colored areas denote plastic deformations, as the stress is equal to the yield stress of steel. Finally, Figure 11 comparatively gives the time histories of equivalent von-Mises stress. Specifically, the measured values at positions SG1-SG4, with the numerically calculated stresses (FE analysis) compared at matching response locations. A direct comparison of these results confirms that the maximum values of the experimentally measured stresses are very close to those computed numerically. More closely, the magenta dashes-dotted line presents the time history of the equivalent von-Mises stresses computed from the analysis of the FE model. Red, blue and green dashed lines present the time histories of von-Mises stresses from the three conducted experimental trials, whereas the black continuous line represents the mean time history of the three trials. Lastly, the red asterisk denoted the maximum value in time from the numerical analysis and the black dot the respective maximum value of the mean time history of measured stresses. A closer examination of this graph clearly validates numerical analysis from the in situ measured data at locations SG2, SG3 and SG4 both in time and in maximum equivalent von-Mises stress. However, although SG1 measurement and 
location matches in maximum stress value, it presents an inconsistency in the time domain variation attributed to faulty location mismatch between experimental set-up and FE model or uncertainties arisen from simplifications during the development of the FE model.

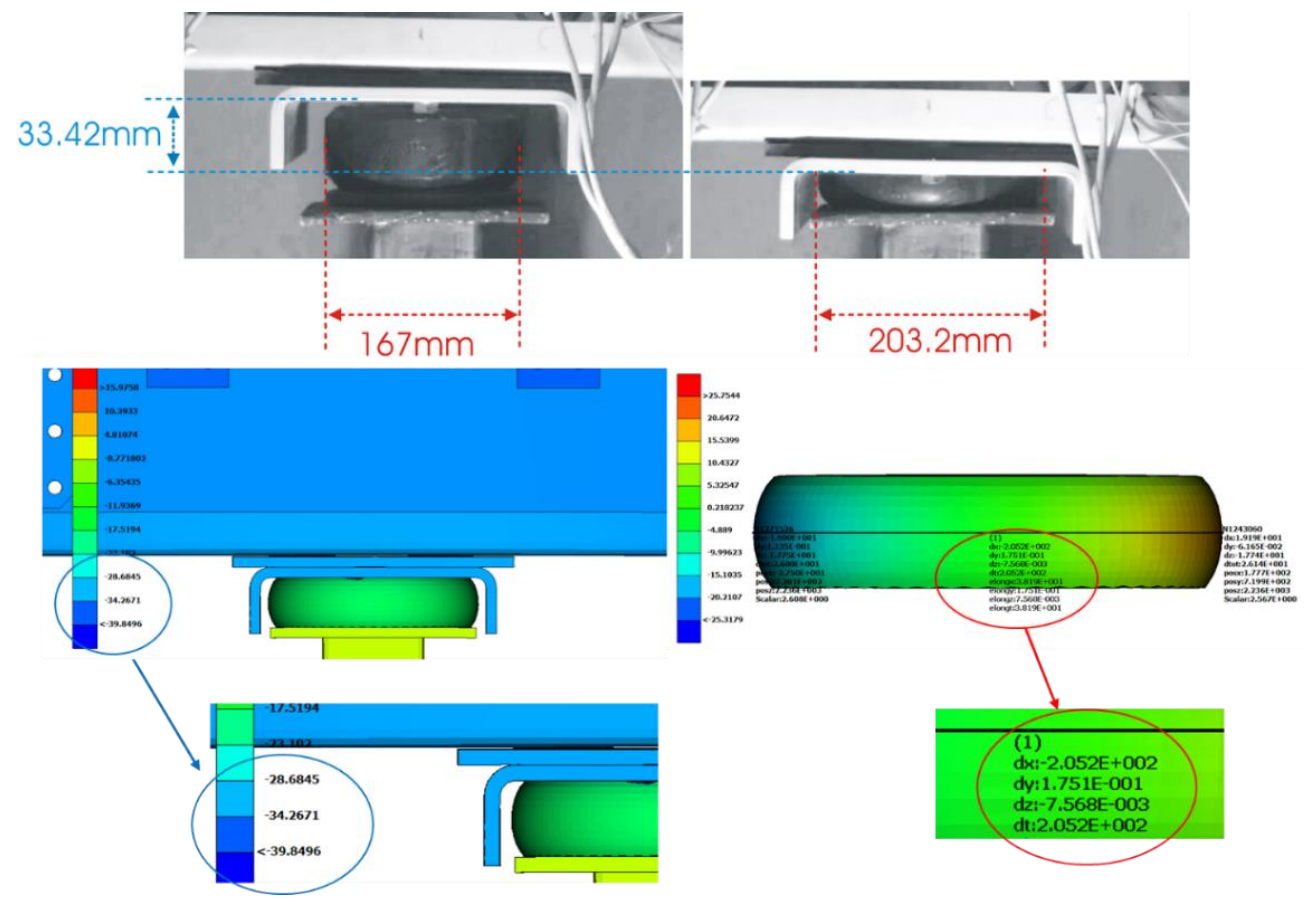

Figure 9. Detailed comparison of the lateral and vertical deformation results at maximum displacement between numerical analysis and experimental measurements.
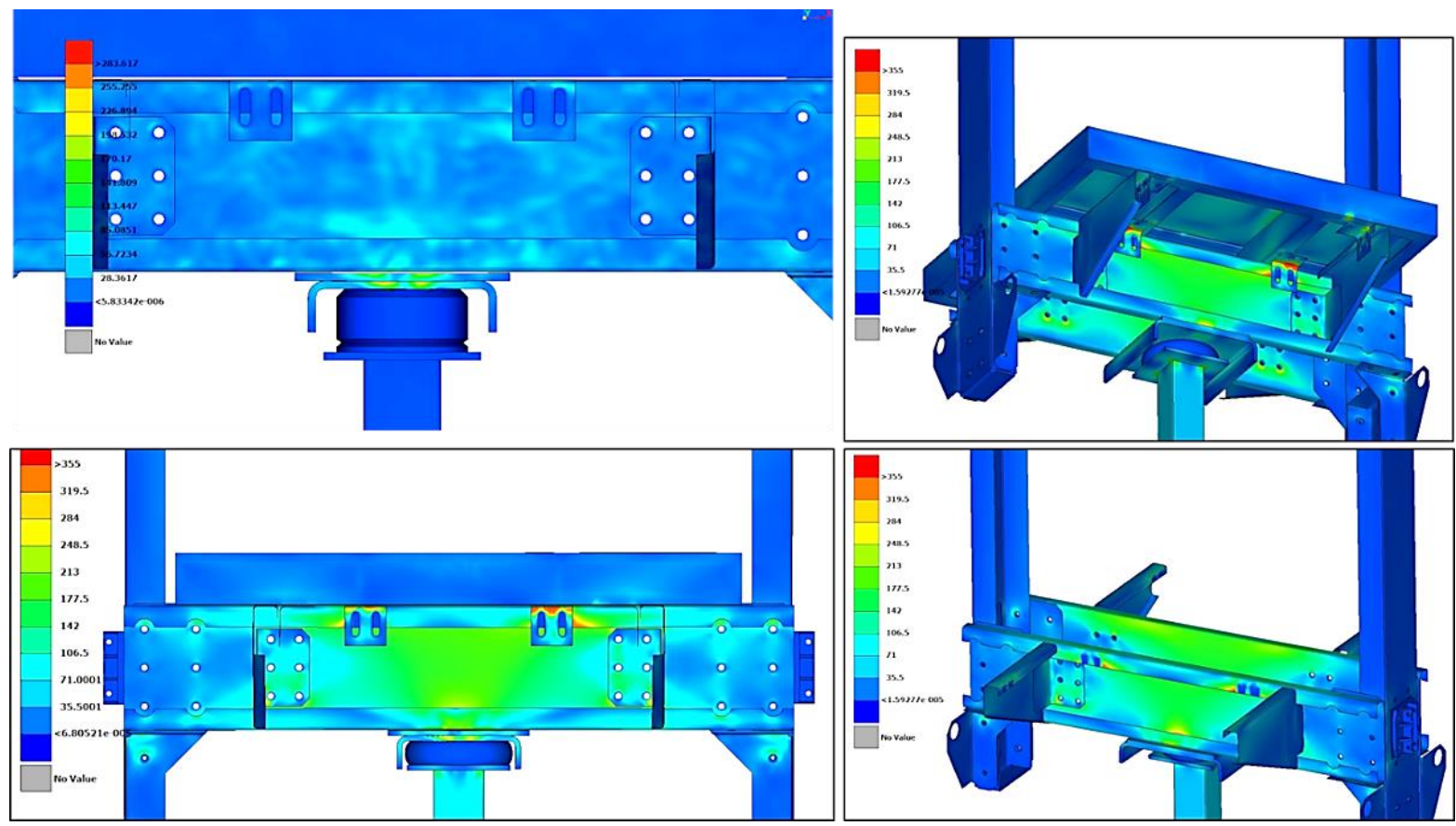

Figure 10. Distribution of equivalent von-Mises stresses on the test-case chassis. 

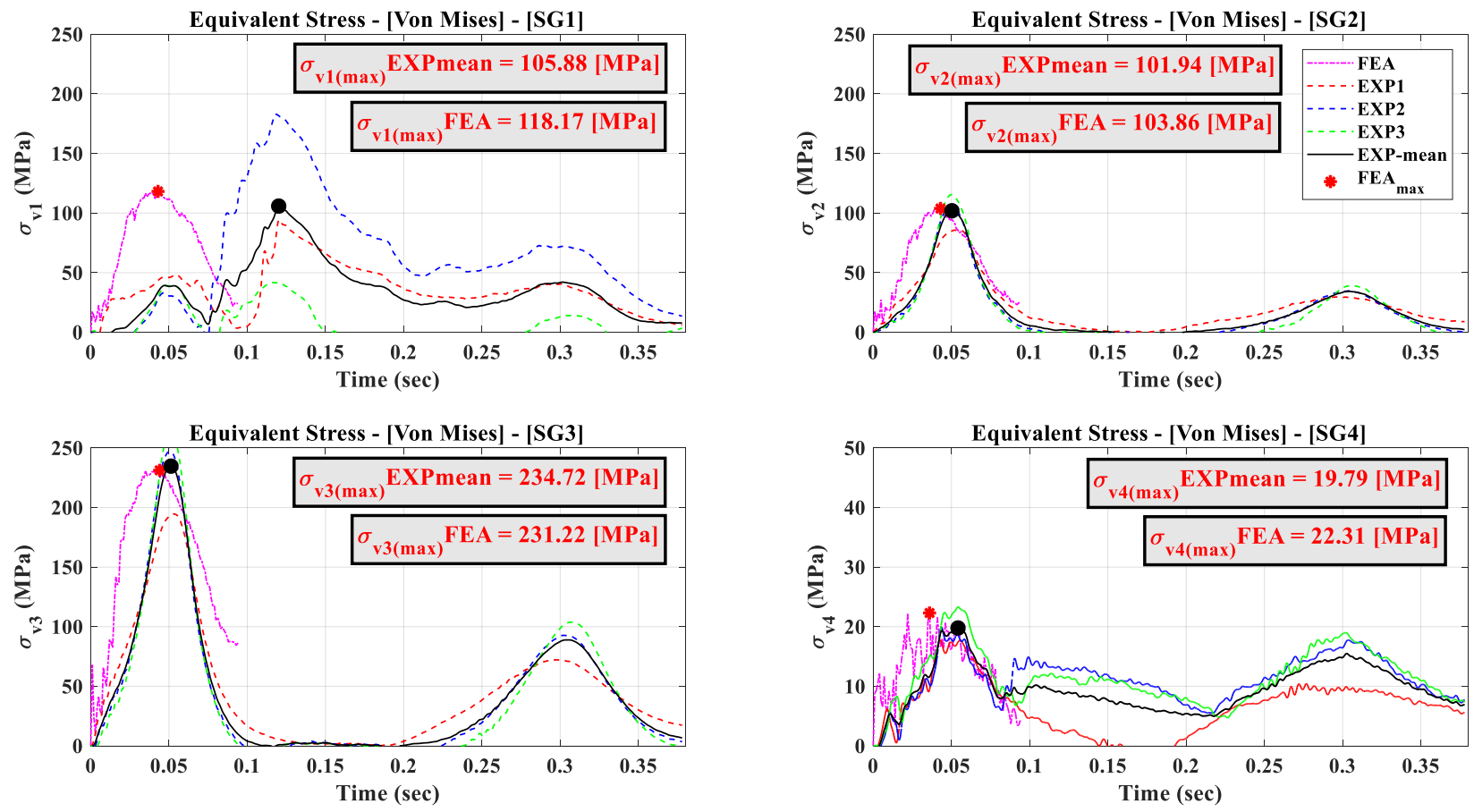

Figure 11. Comparison of experimentally measured (dashed lines) and numerically determined (continuous line) equivalent (von Mises) stress time histories, together with the maximum value of this stress, at the locations (SG1-SG4) during in situ fail scenarios.

The above results, both in maximum displacement, vertical and lateral deformation and in equivalent von-Mises stress, clearly increase confidence of the characterized material model of the shock absorber, producing a high-fidelity FE model of the buffer. Specifically, in order to evaluate and judge the results presented in Figures 9 and 11 one should take into consideration the numerous inevitable approximations and simplifications made in order to develop the large-scale FE model of the test-case elevator chassis and set-up the explicit nonlinear transient response analysis of the crash test scenario, compared to the physical experimental arrangement and free fall phenomenon. In detail, regarding Figure 9 the maximum vertical displacement along with lateral deformation of the absorber as measured using the high-speed camera, was perfectly validated by the numerical predictions despite the above conventions. Furthermore, regarding graphs in Figure 11 matching both maximum stress values and instant of time they appear in three (3) out of four (4) measured locations, i.e., missing one location, is justified by the simplifications of the numerical model as well as the unmodelled but measured environmental interactions. A difference in duration of the analysis $(0.11 \mathrm{~s})$ and the experimental data $(0.35 \mathrm{~s})$ is detected. The only reason is that the main interest is focused in the first impact of the elevator chassis on the shock absorber and not in the following bouncing that occurs until it stops moving, whereas measurements during the experiment were being recorded until the elevator chassis stopped bouncing. The presented results create confidence in relation to the detail and accuracy of the results of the FE model of the elevator chassis, as well as with the FEM fidelity of the updated absorber. Thus, the conclusion of this comparison is that for the size and complexity of the system examined, the differences are considered reasonable, and what is important is that the real structure and numerical model exhibit similar dynamic behavior, which is the goal of this work.

\section{Analysis and Optimal Design of the New-Design Elevator Chassis}

Finally, the detailed FE model of a new-design elevator chassis was developed using nominal steel material properties, where Young's modulus is E $=210 \mathrm{GPa}$, Poisson's ratio $v=0.3$ and density $\rho=7850 \mathrm{~kg} / \mathrm{m}^{3}$ with an elastic-full plastic behavior having a 
yield stress at $\sigma_{\text {yield }}=355 \mathrm{MPa}$. Respectively, the updated parameters of the hyperelastic Mooney-Rivlin material model were assigned to the polyurethane elastomer shock absorber and an explicit nonlinear transient response analysis was carried out within DYNAMIS at a time step of $\Delta \mathrm{t}=10^{-7} \mathrm{~s}$. The geometry of the chassis is discretized using mainly rectangular and triangular shell finite elements. Additionally, solid (hexahedral) elements, rigid body elements and some other special elements are used. The total number of degrees of freedom in the resulting FE models (for the chassis and the cabin) is about $1,600,000$. Appropriate computational method [31], was used in order to estimate the dynamic response of the system, where in the final dynamic analysis set-up, the chassis is fully loaded and is accelerated downwards by gravity $(1 \mathrm{~g})$ in order to simulate accurately the free fall experiment. FE Analysis solver DYNAMIS [21,24] directly uses gravity information in the equations of motion, partitioning and integrating them in accordance with the type of motion specified. The model was then solved numerically in explicit nonlinear transient response analysis in order to calculate displacements, accelerations and maximum stresses developed during the crash on the buffer system.

Based on the results of this analysis the elevator chassis was redesigned and optimized, in order to satisfy ultimate and service limit states, achieving the minimum design stresses and minimum displacements on its flexible parts at an incident of a free-fall crash scenario. As a final step, the new-design elevator chassis was designed and developed, to experimentally validate in real operating conditions the accuracy of the numerical results. Figure 12 presents the FE model of the new-design elevator chassis as it was finally designed before construction.
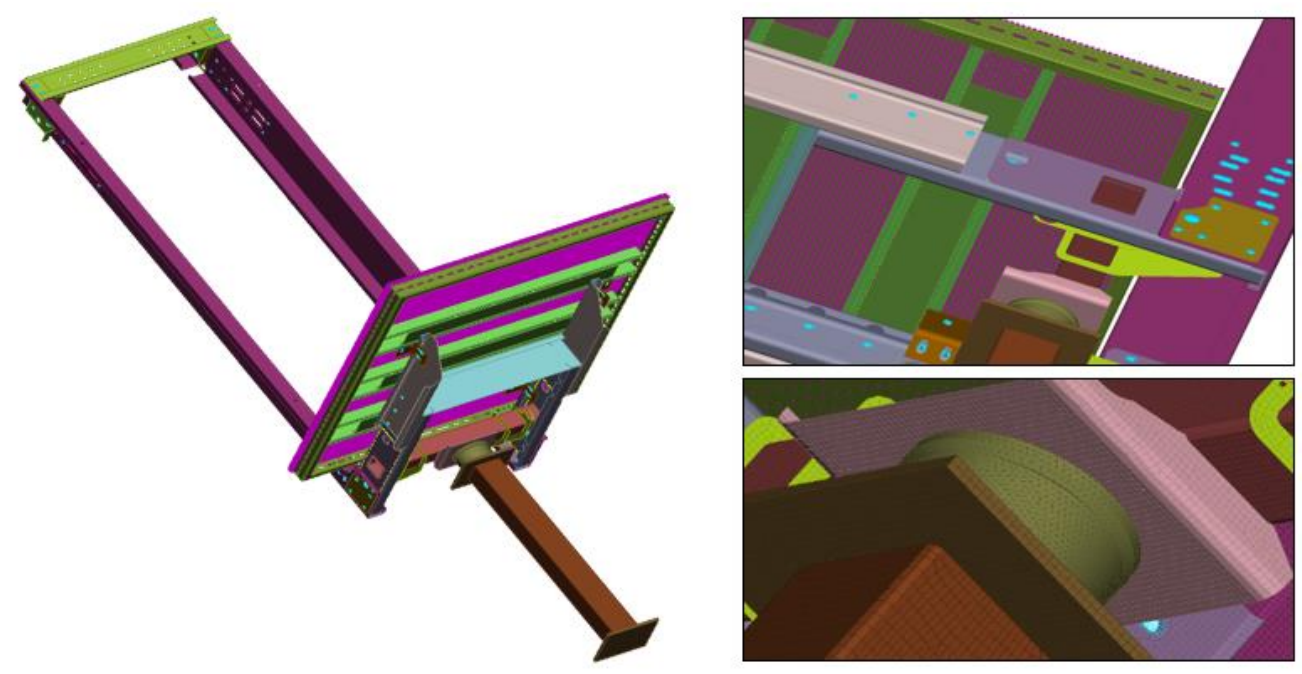

Figure 12. New-design elevator chassis FE model.

Following that, two cases were examined with different thicknesses for the settlement plate, meaning the plate of the new chassis that comes first in contact with the shock absorber. The first case was a settlement plate of $6 \mathrm{~mm}$ thickness and the second case was $10 \mathrm{~mm}$.

At first, Figure 13 presents the whole new-design chassis colored by contours of the equivalent von-Mises stress distribution for the $6 \mathrm{~mm}$ settlement plate thickness. From these results, a plastic deformation of the settlement plate propagated deformation at the suspension beam of the new-design chassis and compromises serviceability with large deformation of the cantilever flexible parts, as presented in Figure 14.

Figure 15 presents the new-design chassis colored by contours of the equivalent vonMises stress distribution for the $10 \mathrm{~mm}$ settlement plate thickness. From these results an elastic deformation of the settlement plate is predicted and no touching on the suspension beam of the new-design chassis is detected, as presented in Figure 15. Finally, serviceability 
limit state is satisfied as the deformation of the cantilever flexible parts were decreased, as presented also in Figure 16.

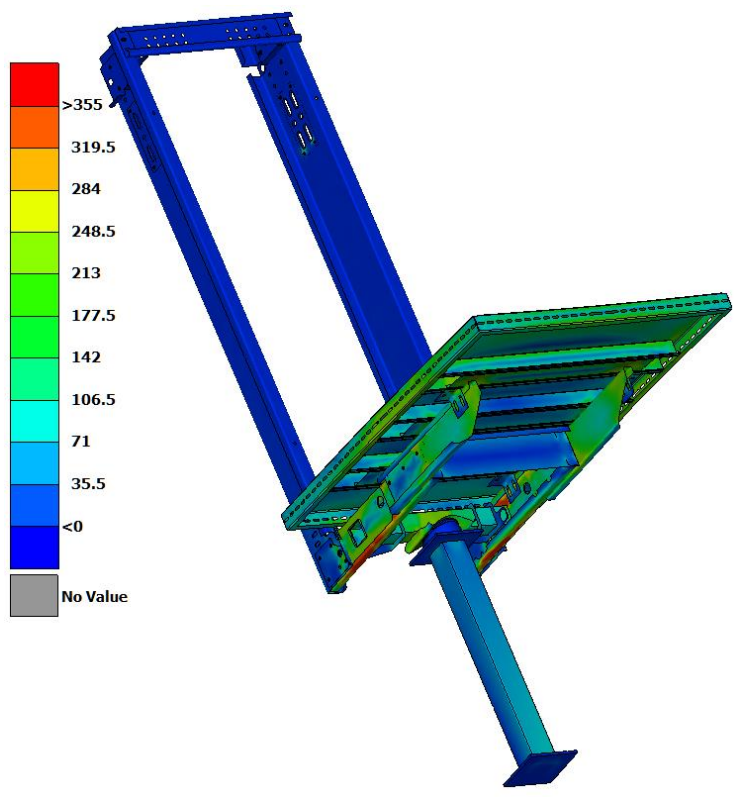

Figure 13. Von-Mises stress distribution on new-design elevator chassis for $6 \mathrm{~mm}$ settlement plate thickness.
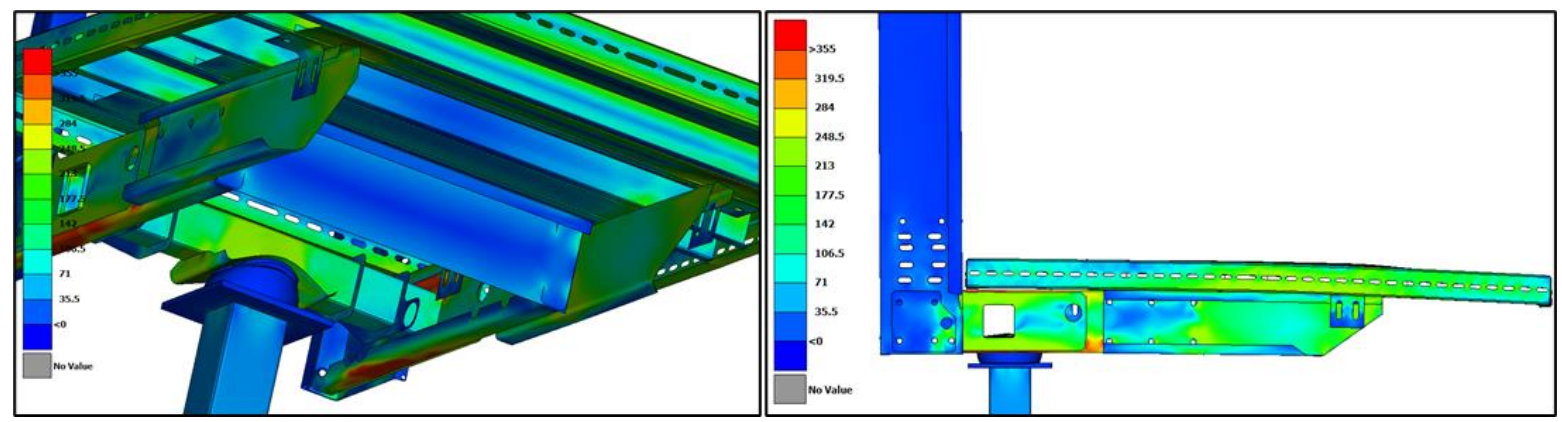

Figure 14. Von-Mises stress distribution on new-design elevator chassis for $6 \mathrm{~mm}$ settlement plate thickness. Plastic deformation of the settlement plate touching suspending beam. Deformation of flexible cantilever components.

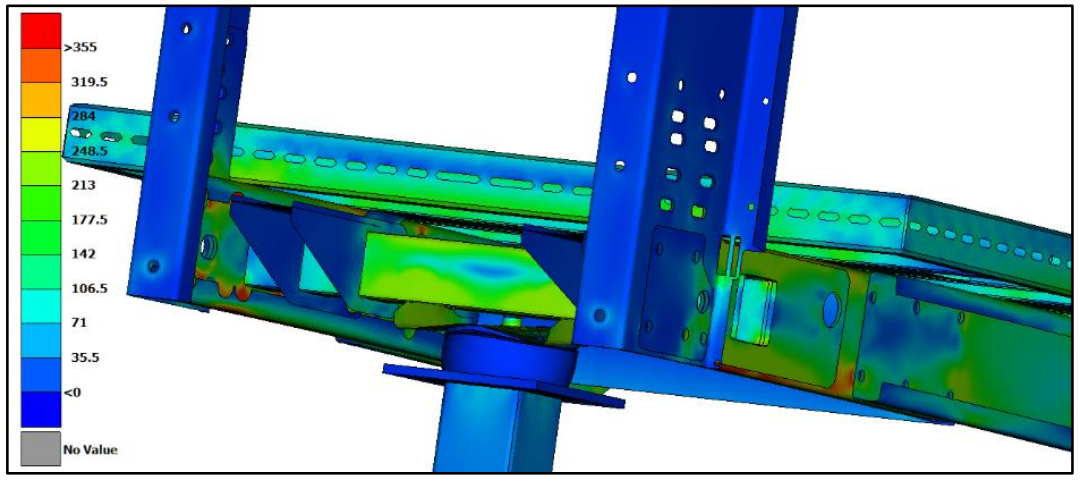

Figure 15. Von-Mises stress distribution on new-design elevator chassis for $10 \mathrm{~mm}$ settlement plate thickness. Elastic deformation of the settlement plate not touching suspending beam. 


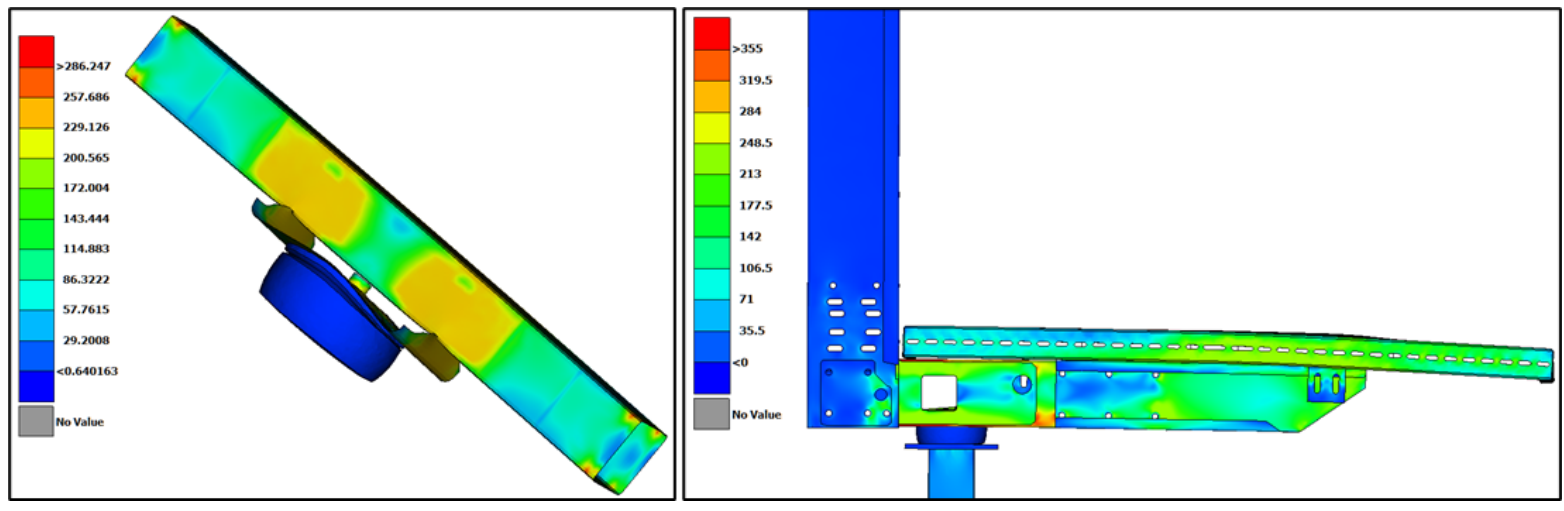

Figure 16. Von-Mises stress distribution on new-design elevator chassis for $10 \mathrm{~mm}$ settlement plate thickness. Detail of the settlement plate and suspending beam. Deformation of flexible cantilever components.

\section{Conclusions}

An optimal design procedure of an elevator chassis crashing on a hyperelastic shock absorber in a fail scenario is presented in this work. At first, specimens of the real rubberlike absorber were tested under uniaxial compression deformations, in a loading machine, extracting experimental stress-strain curves. A discrete Finite Element (FE) model of the examined absorber was developed and a Mooney-Rivlin material model was assigned. Applying a fully parallelizable state-of-the-art stochastic model updating methodology, coupled with robust, accurate and efficient Finite Element Analysis (FEA) software, the hyperelastic behavior of the shock absorber was validated under uniaxial quasi-static loading conditions, in order to tune the material parameters and develop a high-fidelity FE model of the buffer system. Next, triaxial accelerometers and strain gauges were placed at selected critical points of a test-case elevator chassis and a series of experimental measurements was carried out under a real crash scenario, in order to experimentally validate the stress levels developed. Using the updated hyperelastic material properties, defining the nonlinear hyperelastic behavior of the buffer, an explicit nonlinear analysis of the test-case elevator system crashing on the buffer absorber was carried out. Based on the results of this analysis a new-design elevator chassis was redesigned and optimized in order to achieve minimum design stresses and deformations. A direct comparison of the numerical and experimental data validated the reliability and accuracy of the methodology applied.

Author Contributions: Conceptualization, D.G., A.A. and I.C; methodology, D.G. and A.A; software, D.G. and A.A; validation, D.G., A.A. and I.C.; formal analysis, D.G., A.A. and I.C; investigation, D.G. and A.A.; resources, A.A and I.C..; data curation, D.G.; writing-original draft preparation, D.G. and A.A.; writing-review and editing, D.G.; visualization, D.G.; supervision, D.G.; project administration, D.G.; funding acquisition, D.G. and I.C. All authors have read and agreed to the published version of the manuscript.

Funding: This research received no external funding.

Institutional Review Board Statement: Not applicable.

Informed Consent Statement: Not applicable.

Data Availability Statement: All Figures and data created by the authors.

Conflicts of Interest: The authors declare no conflict of interest.

\section{References}

1. Shanks, R.A.; Kong, I. General Purpose Elastomers: Structure, Chemistry, Physics and Performance. In Advances in Elastomers I: Blends and Interpenetrating Networks; Visakh, P.M., Thomas, S., Chandra, A.K., Mathew, A.P., Eds.; Springer: Berlin/Heidelberg, Germany, 2013; pp. 11-45.

2. Flory, P.J. Principles of Polymer Chemistry; Cornell University Press: Ithaca, NY, USA, 1953. 
3. Pouriayevali, H.; Guo, Y.B.; Shim, V.P.W. A visco-hyperelastic constitutive description of elastomer behaviour at high strain rates. Procedia Eng. 2011, 10, 2274-2279. [CrossRef]

4. Patel, R.P.; Shin, J. Compounding and Processing of Plastic/Rubber Blends. In Encyclopedia of Polymer Blends; Isayev, A.I., Ed.; Wiley-VCH: Weinheim, Germany, 2011; Chapter 3; pp. 109-162. Available online: https://onlinelibrary.wiley.com/doi/abs/10.1 002/9783527805242.ch3 (accessed on 30 January 2022).

5. Rivlin, R.S. Torsion of a Rubber Cylinder. J. Appl. Phys. 1947, 18, 444-449. [CrossRef]

6. Soares, R.M.; Gonçalves, P.B. Large-amplitude nonlinear vibrations of a Mooney-Rivlin rectangular membrane. J. Sound Vib. 2014, 333, 2920-2935. [CrossRef]

7. Beda, T. An approach for hyperelastic model-building and parameters estimation a review of constitutive models. Eur. Polym. J. 2014, 50, 97-108. [CrossRef]

8. Hao, D.; Li, D.; Liao, Y. A finite viscoelastic constitutive model for filled rubber-like materials. Int. J. Solids Struct. 2015, 64-65, 232-245. [CrossRef]

9. Sasso, M.; Palmieri, G.; Chiappini, G.; Amodio, D. Characterization of hyperelastic rubber-like materials by biaxial and uniaxial stretching tests based on optical methods. Polym. Test. 2008, 27, 995-1004. [CrossRef]

10. Dall'Asta, A.; Ragni, L. Nonlinear behavior of dynamic systems with high damping rubber devices. Eng. Struct. 2008, 30, 3610-3618. [CrossRef]

11. Spottswood, S.M.; Allemang, R.J. On the Investigation of Some Parameter Identification and Experimental Modal Filtering Issues for Nonlinear Reduced Order Models. Exp. Mech. 2007, 47, 511-521. [CrossRef]

12. Arailopoulos, A.; Giagopoulos, D. Nonlinear constitutive force model selection, update and uncertainty quantification for periodically sequential impact applications. Nonlinear Dyn. 2020, 99, 2623-2646. [CrossRef]

13. Hadjidoukas, P.E.; Angelikopoulos, P.; Papadimitriou, C.; Koumoutsakos, P. П4U: A high performance computing framework for Bayesian uncertainty quantification of complex models. J. Comput. Phys. 2015, 284, 1-21. [CrossRef]

14. Hansen, N. The CMA Evolution Strategy: A Tutorial. 2011, Research centre Saclay-^Ile-de-France Universite' Paris-Saclay, LRI Available online: http:/ / www.cmap.polytechnique.fr/ \{\}nikolaus.hansen/cmatutorial110628.pdf (accessed on 4 March 2022).

15. Hansen, N. The CMA Evolution Strategy A Comparing Review. Towards A New Evol. Comput. 2006, 192, 75-102.

16. Hansen, N.; Müller, S.D.; Koumoutsakos, P. Reducing the Time Complexity of the Derandomized Evolution Strategy with Covariance Matrix Adaptation (CMA-ES). Evol. Comput. 2003, 11, 1-18. [CrossRef] [PubMed]

17. Giagopoulos, D.; Arailopoulos, A. Computational framework for model updating of large scale linear and nonlinear finite element models using state of the art evolution strategy. Comput. Struct. 2017, 192, 210-232. [CrossRef]

18. Zapico-Valle, J.L.; Alonso-Camblor, R.; González-Martínez, M.P.; García-Diéguez, M. A new method for finite element model updating in structural dynamics. Mech. Syst. Signal Processing 2010, 24, 2137-2159. [CrossRef]

19. Giagopoulos, D.; Arailopoulos, A.; Chatziparasidis, I.; Sapidis, N.S. Optimum design of large-scale systems considering material nonlinearities and uncertainties. Comput. Struct. 2019, 223, 106102. [CrossRef]

20. Giagopoulos, D.; Arailopoulos, A.; Natsiavas, S. A model-based fatigue damage estimation framework of large-scale structural systems. Struct. Health Monit. 2019, 20, 834-884. [CrossRef]

21. DTECH, DYNAMIS 3.1.1.; DTECH: Thessaloniki, Greece, 2013.

22. Hadjidoukas, P.E.; Angelikopoulos, P.; Voglis, C.; Papageorgiou, D.G.; Lagaris, I.E. NDL-v2.0: A new version of the numerical differentiation library for parallel architectures. Comput. Phys. Commun. 2014, 185, 2217-2219. [CrossRef]

23. Hadjidoukas, P.E.; Lappas, E.; Dimakopoulos, V.V. A Runtime Library for Platform-Independent Task Parallelism. In Proceedings of the 20th Euromicro International Conference on Parallel, Distributed and Network-Based Processing, Garching, Germany, 15-17 February 2012.

24. DYNAMIS 3.1.1 Solver Reference Guide; DTECH: Thessaloniki, Greece, 2013.

25. Mathworks, Matlab; Mathworks Inc.: Natick, MA, USA, 2016.

26. Saccomandi, G.; Vergori, L. Generalised Mooney-Rivlin models for brain tissue: A theoretical perspective. Int. J. Non-Linear Mech 2019, 109, 9-14. [CrossRef]

27. Mooney, M. A Theory of Large Elastic Deformation. J. Appl. Phys. 1940, 11, 582-592. [CrossRef]

28. Mullins, L. Effect of stretching on the properties of rubber. Rubber Chem. Technol. 1948, 21, 281-300. [CrossRef]

29. Mars, W.V. Evaluation of a Pseudo-Elastic Model for the Mullins Effect. Tire Sci. Technol. 2004, 32, 120-145. [CrossRef]

30. BETA CAE Systems, S.A. ANSA \& META-Post; BETA CAE Systems, S.A.: Thessaloniki, Greece, 2018.

31. Giagopoulos, D.; Chatziparasidis, I. Optimum design, finite element model updating and dynamic analysis of a full laminated glass panoramic car elevator. In Proceedings of the 7th European Congress on Computational Methods in Applied Sciences and Engineering, ECCOMAS Congress, Crete Island, Greece, 5-10 June 2016; National Technical University of Athens: Athens, Greece, 2016.

32. Blatz, P.J.; Ko, W.L. Application of finite elastic theory to the deformation of rubbery materials. Trans. Soc. Rheol. 1962, 6, 223-252. [CrossRef]

33. Bolzon, D.G.; Vitaliani, R. The Blatz-Ko material model and homogenization. Arch. Appl. Mech. 1993, 63, 228-241. [CrossRef] 